\title{
La argumentación como estrategia para el desarrollo de competencias fundamentales en docentes en formación
}

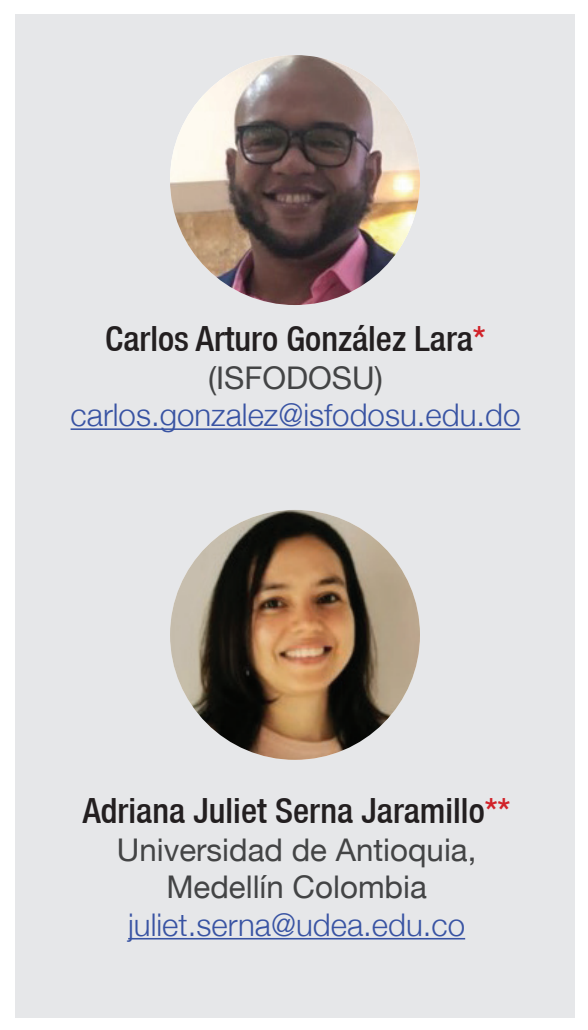

Argumentation as a strategy for
the development of fundamental
competences in teachers in training

Recibido: 2 de noviembre de 2021 | Aprobado: 23 de noviembre de 2021

\section{Resumen}

El presente artículo describe las concepciones de algunos docentes del ISFODOSU acerca de la argumentación como estrategia para el desarrollo de las competencias fundamentales en la formación de maestros. El diseño metodológico se centra en un enfoque mixto, de carácter descriptivo. Se diseñó y aplicó un cuestionario semiestructurado a 31 docentes, de cinco recintos de dicha institución. El análisis de los resultados permitió evidenciar la comprensión de la estrategia de argumentación, destacando que constituye un proceso complejo, pues requiere el desarrollo de un pensamiento lógico, crítico y capaz de utilizar las evidencias disponibles, proceso asociado a la comunicación, al razonamiento y justificación. Como conclusión, los datos evidencian que los docentes valoran positivamente la estrategia de argumentación porque les permite promover en los estudiantes el desarrollo de la habilidad para defender una postura y resolver problemas a partir del razonamiento de las situaciones sustentado en evidencias y elementos teóricos de fuentes confiables de información.

Palabras clave: Argumentación, competencias fundamentales, concepciones, estrategias de argumentación y formación docente.

\footnotetext{
Magister en Psicodidáctica.Coordinador de la División de Extensiones. Maestro a tiempo completo de Práctica Docente y Pasantía en el Instituto Superior de Formación Docente Salomé Ureña, Recinto Urania Montás (ISFODOSU). Para contactar al autor: carlos.gonzalez@isfodosu.edu.do ORCID https://orcid.org/0000-0002-6159-9710

** Doctora en Neuropsicología clínica por la Universidad de Salamanca. Magíster en Neuropsicología y Educación en UNIR. Licenciada en Educación Especial por la Universidad de Antioquia. Profesora por asignatura y Coordinadora de prácticas de la Licenciatura en Educación Infantil de la Facultad de Educación de la Universidad de Antioquia, Medellín, Colombia. Para contactar a la autora: juliet.serna@udea.edu.co ORCID: https://orcid.org/00000001-6166-049X
}

ISSN (en línea): 1814-4152 / Sitio web: http://cuaderno.pucmm.edu.do

Cómo CITAR: González-Lara, C. y Serna-Jaramillo, A. (2022). La argumentación como estrategia para el desarrollo de competencias fundamentales en docentes en formación. Cuaderno de Pedagogía Universitaria, 19 (37), 133-143. 


\section{Abstract}

This article describes the conceptions of some ISFODOSU teachers about argumentation as a strategy for the development of fundamental competencies in teacher training. The methodological design focuses on a mixed, descriptive approach. A semi-structured questionnaire was designed and applied to 31 teachers from five campuses of said institution. The analysis of the results made it possible to demonstrate the understanding of the argumentation strategy, highlighting that it constitutes a complex process, since it focuses on the analysis of information, case studies, debates, process associated with communication and reasoning and justification. In conclusion, the data show that teachers value the argumentation strategy positively because it allows them to promote in students the development of the ability to argue and solve problems based on the reasoning of situations and support through the data and theoretical elements of reliable sources of information.

Keywords: argumentation, fundamental competences, conceptions, argumentation strategies and teacher training.

\section{Introducción y marco referencial}

El enfoque por competencias en el Ministerio de Educación Superior Ciencia y Tecnología (MESCYT), es adoptado y promovido a partir del establecimiento de la ordenanza 09-2015, la cual motivó a todas las instituciones de educación superior a regirse por la misma, en este sentido, se realizó una transformación curricular de todos los planes y programas de estudio, así como la actualización del enfoque de enseñanza de los formadores (Santana Soriano, 2020). En el Instituto Superior de Formación Docente Salomé Ureña (ISFODOSU) se adoptó el enfoque, formalizando 5 competencias fundamentales articuladas a las propuestas en dicha ordenanza, las cuales motivó a desarrollar en sus docentes en formación desde las distintas carreras que esta promueve y a sus docentes formadores a crear las condiciones para que estas puedan ser desarrolladas a partir de los cambios en los procedimientos, las formas y maneras de enseñar.

En este sentido, se llevó a cabo esta investigación con el fin de conocer las concepciones y práctica del profesorado acerca de la argumentación como estrategia para el desarrollo de las competencias fundamentales en la formación docente, intentando responder a las siguientes interrogantes: ¿Cuáles son las concepciones, ideas, creencias y pensamientos que tienen los docentes acerca de la argumentación como estrategia para el desarrollo de competencias fundamentales en docentes en formación?, ¿cuál es el nivel de importancia de esta estrategia en su práctica docente?, y ¿cuáles son las ventajas que esta aporta a los docentes en formación?

Por lo que esta investigación tiene como objetivos identificar las concepciones, ideas, conocimientos y creencias que tiene el profesorado del ISFODOSU acerca de la argumentación como estrategia para el desarrollo de las competencias fundamentales en la formación docente; cuantificar el nivel de importancia que le otorgan los docentes y exponer las creencias e ideas que poseen los profesores frente a las ventajas de esta estrategia en su práctica.

Fundamentación teórica competencias fundamentales

Las competencias fundamentales en el nivel superior suponen la capacidad efectiva para desarrollar de forma eficaz una determinada actividad profesional o laboral, lo que exige disponer de un conjunto de aptitudes y recursos intelectuales, actitudes sociales y valores para afrontar eficientemente determinadas situaciones profesionales (Tobón, 2006; Zabalza y Beraza, 2003). Por ello, es necesario que los estudiantes actúen de manera reflexiva y crítica. También, sugiere que desde los procesos de enseñanza se ponga en práctica la integración de saberes, habilidades, destrezas; así como el reconocimiento e identidad del sujeto en relación con sus actitudes, aptitudes y valores. 
Cabe destacar que el enfoque de competencias nace, según Ruiz (2009), debido al replanteamiento y organización de la educación como un proceso de facilitación de aprendizaje y por la posibilidad de formar profesionales capaces de resolver problemas de sus contextos de manera eficaz y tomando en cuenta la articulación de sus saberes con la práctica. Por ello, son procesos complejos de desempeño, que focalizan tres aspectos específicos, primero la integración de los conocimientos, los procesos cognitivos, las habilidades y los valores ante los problemas; segundo, el diseño de programas de formación con base en los requerimientos contextuales; y, por último, la orientación de la educación por medio de estándares e indicadores de calidad en todos sus procesos.

Sin embargo, Tobón (2006), sugiere el cambio en la manera de enseñanza tradicional centrada en la adquisición y transmisión de conocimientos impartida por los docentes, por un proceso dinámico, donde el docente pasa a ser el orientador y guía del proceso de enseñanza y aprendizaje, mientras los alumnos asumen todo el rol protagónico (Sanz y Pedreño, 2011). A su vez, esto les insta a que participen de procesos formativos y se nutran especialmente de una serie de competencias y estrategias (Zabalza y Beraza, 2003). Por lo tanto, el estudiante se convierte en el protagonista de sus experiencias, las cuales le facilitan el aprendizaje, no solo de los contenidos de las distintas disciplinas que reciben, sino también que se deben tomar en cuenta para su desarrollo, así como los aportes significativos que estas les proporcionan (Organización para la Cooperación y Desarrollo Económico, OCDE, 2006).

Es en este sentido, el modelo de competencia en el nivel de educación superior asume que los docentes en formación desarrollen el pensamiento crítico y reflexivo en su proceso formativo, manifestando todas sus capacidades y destrezas combinadas con el conocer (curiosidad), saber (conocimientos), ser (habilidades) y convivir con los demás, donde sean capaces de interactuar y dar opiniones sustentadas en argumentos válidos a partir de las pruebas disponibles.
Formación inicial de maestros

Según el artículo 2 de la Ordenanza 5-2004 que establece el Reglamento del Instituto Nacional de Formación y Capacitación del Magisterio (INAFOCAM), la formación centrada en el ámbito docente es el:

"conjunto de experiencias y procesos diversos que posibilitan adquirir las competencias adecuadas que requieren los distintos actores educativos, en sus diferentes roles y etapas dentro de su desempeño, todo ello concebido y articulado bajo los paradigmas y enfoques asumidos por el Sistema Educativo Dominicano" (INAFOCAM, 2004, p.14).

Esta formación es un proceso permanente, dinámico, integrado, multidimensional en el que convergen, entre otros elementos, la disciplina y sus aspectos teóricos, metodológicos, epistemológicos, didácticos, psicológicos, sociales, filosóficos e históricos, para lograr la profesionalización de la docencia (Chehaybar- Kuri y Amador-Bautista, 2003).

Para Córdoba et al. (2005), citando a González (1995), la formación inicial "supone la etapa formativa anterior al desempeño de la profesión docente, encaminada a favorecer y desarrollar las capacidades, disposiciones y actitudes de los profesores, con el fin de prepararles y facilitarles la realización eficaz de su tarea" (p. 216).

En la actualidad, hablar de la formación inicial de docentes supone hablar de calidad (Levine, 2006). Por ello, esta se encarga de preparar profesionalmente a los estudiantes para la docencia, insertándose en el ámbito pedagógico a través de programas específicos que habilitan y certifican su condición de docentes ante la sociedad (SalazarGómez y Tobón, 2018, citando a Sánchez, 2013).

La formación inicial universitaria constituye el primer requerimiento formal para ingresar a la carrera docente (Ministerio de Educación de la República Dominicana, MINERD, 2016). Por esto, actualmente para ejercer como maestro en el aula es obligatorio formarse en la universidad como profesional de la enseñanza, establecido en la ordenanza 5-2004.

- En la República Dominicana existen más de 25 instituciones de educación superior dedicadas a la formación inicial 
de docentes, dentro de estas, el Instituto de Superior de Formación Docente Salomé Ureña, ISFODOSU, la cual se dedica exclusivamente a formar docentes. Esta toma en cuenta y pone en práctica el enfoque por competencias en todos sus planes de estudios priorizando las necesidades de los estudiantes, los contextos actuales que los componen, las metodologías de enseñanza y lo que exige la sociedad actual, vinculado a lo que establece la Normativa 09-2015 del Ministerio de Educación Ciencia y Tecnología, MESCYT. En relación con las competencias fundamentales que adoptó, y las cuales están presentes en el Plan de estudio de Educación Primaria, Segundo Ciclo, páginas 4 y 5, se encuentran:

- Desarrollo intra e interpersonal, la cual establece relaciones efectivas consigo mismo y con los demás de manera respetuosa y responsable en diversos contextos.

- Sociocultural, la cual valora la cultura local, nacional y global mostrando respeto y aceptación ante la diversidad.

- Profesional, centrándose en las aptitudes creativas, de compromiso y rigor científico para desempeñar su labor pedagógica como agente efectivo del proceso de aprendizaje.

- Comunicativa, que promueve las habilidades comunicativas que le permiten actuar adecuadamente con diferentes actores en diversos contextos, utilizando los medios de comunicación disponibles.

- Pensamiento reflexivo y crítico, la cual permite desarrollar una conciencia crítica orientada a la emancipación y autonomía para la mejora de la experiencia individual, colectiva y mundial.

Es preciso mencionar que para el cumplimiento de estas competencias es necesario que el formador de formadores implemente diversas y nuevas metodologías activas (estrategias, técnicas, actividades de enseñanza y evaluación, actividades y recursos) que favorezcan la creación de espacios de reflexión, la capacidad para presentar su punto de vista y defender posturas, aprendizajes significativos, el desarrollo de competencias cognitivas, así como la práctica acompañada que les permita avanzar en la autogestión y autorregulación de los conocimientos.

\section{La argumentación como estrategia}

Las estrategias de enseñanza son procedimientos generales que el docente diseña, selecciona y emplea para desarrollar sus procesos pedagógicos. Para González y Pérez (2012), son un conjunto de actividades sistemáticamente organizadas y planificadas con la finalidad de encontrar respuestas y solucionar posibles problemáticas. Según el Ministerio de Educación de la República Dominicana, MINERD (2016), estas son un conjunto de procedimientos diseñados y realizados por los docentes, con una intencionalidad pedagógica clara, coherente, precisa y explícita, y se apoya en las técnicas para su concreción. Su importancia radica en potenciar el espacio para que se genere el aprendizaje y se desarrollen las competencias, por tal razón, deben incluirse acorde con las características de aprendizaje de los diferentes grupos de estudiantes y enfocadas en los procesos de comunicación, pensamiento lógico, la observación de la naturaleza y su entorno social (MESCYT, 2015). Las cuales les permiten a los estudiantes indagar en diversas fuentes, analizar problemas, reflexionar sobre su accionar para responder a las problemáticas de sus contextos, argumentar sus ideas basadas en datos, resolver problemas, entre otros elementos relacionados con el desarrollo de sus capacidades, habilidades y destrezas.

Una estrategia considerada importante para el desarrollo de competencias es la de argumentación, reconocida como uno de los fundamentos de la vida en sociedad, ya que orienta las actividades del ser humano y de su cultura e influye de manera determinante en las diversas actividades de la vida (Serrano, 2008, citando a Del Caño, 1999), ya que, como práctica social, la argumentación le permite a la persona actuar ante las discrepancias y conflictos, asumiendo una actitud crítica ante la manipulación de discursos sociales para así establecer y mantener relaciones en el seno del grupo al cual pertenece y al cual debe integrarse (Martínez, 1999). Anteriormente, la argumentación, según Buitrago et al. (2013), citando a Plantin 
(2012), formaba parte de la lógica o "arte de pensar correctamente", de la retórica o "arte de hablar bien" y de la dialéctica o "arte de dialogar bien" ( $p$. 1), supuestos que se extendieron hasta finales de siglo XIX y permitieron la evolución del concepto de argumentación de los filósofos griegos en su manera de ver esta como solo la actividad de razonar, inferir, incidir y argumentar para convencer desde los discursos sociales. Toulmin (1958) la describe como la "constelación de enunciados ligados en un sistema, que otorga al discurso una forma de racionalidad" en su obra The Uses of Argument en que propone su modelo argumentativo, el cual se centra en las razones y argumentos que se pueden dar basado en los datos disponibles, modelo que, según sus pasos, puede ser aplicado en cualquier disciplina u/o actividad (Rodríguez, 2004). De ahí que, la argumentación supone una actividad verbal, social y racional dirigida a convencer de manera razonable y lógica con argumentos apoyados y sustentados en datos (Osborne, 2012). Por ello, esta consiste en la evaluación del conocimiento a partir de las pruebas disponibles (JiménezAleixandre y Puig, 2010).

En el caso de la argumentación como estrategia, es un procedimiento discursivo que, de modo intencional y consciente, utiliza el hablante o el escritor para incrementar la eficacia de su discurso al convencer o persuadir al destinatario en una situación comunicativa donde exista argumentación (Cros, 2003). Esta, en la cultura científica, es considerada como práctica científica de argumentación (Crujeiras y Jiménez Aleixandre, 2012). Sin embargo, para el desarrollo de otras áreas del conocimiento y promoción de competencias, la argumentación es empleada como un gran procedimiento o estrategia pedagógica innovadora (Gallego y Márquez, 2018).

Investigaciones como la de Aldana (2014) precisan que la argumentación como estrategia cumple un rol fundamental en el proceso de enseñanza y guía de la acción educativa, ya que esta genera espacios de comunicación entre pares, entre el educador y el educando, así como diálogos interactivos que facilitan la tarea del profesor en cuanto a la mediación e interacción en el proceso docente educativo. Además de que esta permite ver la capacidad que tienen los estudiantes para argumentar, confrontar y justificar los procesos relacionados con una actividad que implica el desarrollo del pensamiento lógico, reflexivo y crítico. En su conclusión destaca que la argumentación es una forma de comunicación y diálogo para evaluar, definir y estimular producciones y que esta será eficaz y podrá utilizarse como estrategia si se explican las actividades del proceso que implican este tipo de interacción comunicativa.

Para González (2015), esta estrategia como procedimiento en el aula se promueve a partir de la participación activa en debates y foros en donde exponen sus puntos de vista, exposiciones de investigaciones realizadas; intervención de expertos, trabajos en equipo centrados en socializaciones autodirigidas, así como la puesta en práctica de argumentos de forma razonable y lógica partiendo de problemas o situaciones contextualizadas y en las que se motive la indagación y el pensamiento lógico, entre otras habilidades.

Según Buitrago et al. (2013), la importancia de esta estrategia radica en reconocerla como una habilidad cognitivo- lingüística y que debe ser asumida en todas las áreas del conocimiento sustentadas en los planes y programas de estudios, pues el valor dialógico y racional de la argumentación es beneficioso para la construcción de conocimientos. En este sentido, autores como Osborne (2012) y Díaz de Bustamante y Jiménez Aleixandre (1999) sugieren que el profesorado debe diseñar y crear las condiciones para que los estudiantes participen en discursos argumentativos en el que puedan dar respuestas apropiadas o hacer juicios sobre problemas de su contexto. Planteamiento que exige entonces que se ponga en práctica el desarrollo de los procesos cognitivos de indagación, análisis, reflexión, evaluación y síntesis.

Tomando en cuenta lo descrito en esta fundamentación teórica sobre la importancia de la formación de maestros bajo el enfoque por competencias en la República Dominicana, la calidad de la educación en la formación docente y lo que supone la estrategia de argumentación para el desarrollo de las habilidades comunicativas, se plantea identificar las concepciones de los formadores de docentes en formación acerca de la argumentación como estrategia para el desarrollo de las competencias fundamentales en la formación docente. Este estudio cobra mayor importancia ya que en el ISFODOSU hasta el momento no se 
han desarrollado investigaciones con relación al tema destacado, lo que sugiere la actualización y transformación de las prácticas de los docentes formadores y docentes en práctica.

\section{Metodología}

El estudio se desarrolló bajo un enfoque mixto, el cual sustenta los procesos de análisis cualitativos y cuantitativos. Es de tipo descriptivo, no experimental y de carácter transversal. La muestra seleccionada para la presente investigación estuvo conformada por 34 docentes de 5 Recintos del ISFODOSU. En cuanto al rango de edad, 4 docentes se ubican entre 25 a 35 años, 9 docentes entre 35 y 45 años, 16 docentes entre 45 y 55 años, 5 docentes con más de 55 años, de los cuales 8 tienen título de doctorado, 23 de maestría y 3 con licenciatura, los mismos se encuentran organizados en 6 grandes áreas a partir de las asignaturas que imparten, tales como; Psicopedagogía, Práctica Docente, Educación Física, Matemática, Biología y Química e Historia, Ciencias Sociales y Lengua Española.

Para el proceso de recolección de la información se utilizó un cuestionario mixto con ítems de selección múltiple, escala tipo Likert de 5 puntos, preguntas abiertas y análisis de casos. El instrumento partió de la versión del cuestionario sobre concepciones y prácticas acerca de la indagación y argumentación en la enseñanza de las ciencias (CPCIAEC), el cual fue diseñado y aplicado en la investigación de González (2015). Para su aplicación todos los participantes firmaron un consentimiento informado y accedieron de manera voluntaria a hacer parte de la investigación respondiendo el cuestionario.

Para llevar a cabo el proceso de validación del cuestionario, se compartió a través de correo electrónico el instrumento a 5 expertos en diferentes áreas académicas, tales como, psicología, investigación, filosofía y español, con la finalidad de que pudieran revisarlo en relación a los siguientes criterios: pertinencia, claridad y relevancia. Para determinar la validez de contenido, se aplicó la concordancia de jueces a través de la técnica de proporción de acuerdos. Posteriormente, para determinar el nivel de confiabilidad del instrumento, le fue aplicado el cuestionario a 31 docentes; con los datos obtenidos, se aplicó la prueba del Alpha de Cronbach dando como resultado un nivel de fiabilidad de 0.873 para la escala total. Por lo tanto, de acuerdo con los criterios de esta prueba, se determina que el cuestionario a usar es consistente, por lo que la información obtenida es fiable.

Los resultados fueron analizados de manera cualitativa y cuantitativa. Para el análisis cualitativo se utilizó el software Atlas.ti., con el cual se codificaron y analizaron las categorías relacionadas con los objetivos de la investigación. En estos códigos se consideró la frecuencia de los enraizamientos (E), que corresponde al número de citas en que un código aparece y la densidad de los códigos (D), que representa el número de conexiones con otros códigos en las redes semánticas. Para este estudio se realizó la sistematización de los datos en una tabla

Una vez analizados los resultados, se procedió a realizar la discusión y conclusiones contrastando con diferentes fuentes teóricas e investigaciones que permitieron lograr las reflexiones frente a los hallazgos derivados de la presente investigación.

\section{Resultados}

Los resultados y análisis que se presentan a continuación, se organizaron en función de los objetivos planteados.

En relación al primer objetivo sobre las concepciones, ideas, conocimientos y creencias del profesorado del ISFODOSU acerca de la argumentación como estrategia para el desarrollo de las competencias fundamentales en la formación docente, se evidenció que la argumentación para esta población supone un proceso complejo basado en la búsqueda y presentación de datos y pruebas que promueve el trabajo en equipo; es un proceso asociado a la comunicación y de análisis de información. Estas categorías a su vez se conectan con la indagación y lo que esta implica y otros aspectos, tales como: la búsqueda de información en diversas fuentes confiables y procesos de reflexión que permitan validar y argumentar el trabajo colaborativo y de equipo centrado en la interacción y reflexión compartida y la participación en debates, procesos de reflexión, análisis de casos y socialización de contenidos expresados en juicios en los que evidencia su capacidad para razonar, justificar y demostrar los hechos para convencer. (Ver Tabla 1) 
Tabla 1.

Categorías descritas y cantidad de docentes que las eligieron.

\begin{tabular}{|c|c|c|}
\hline $\begin{array}{c}\text { Código y cantidad de docentes en } \\
\text { elegir estas categorías }\end{array}$ & Categorias & Descripción \\
\hline PC (13) & Proceso complejo (PC) & $\begin{array}{c}\text { Implica que la argumentación no es un proceso } \\
\text { sencillo y que además esta requiere realizar } \\
\text { indagaciones }\end{array}$ \\
\hline BPDP (21) & $\begin{array}{c}\text { Búsqueda y } \\
\text { presentación de datos y } \\
\text { pruebas (BPDP) }\end{array}$ & $\begin{array}{c}\text { Esta categoría se refiere a realizar indagaciones en } \\
\text { diversas fuentes confiables y procesos de reflex- } \\
\text { iones que permitan validar y argumentar. }\end{array}$ \\
\hline TE (21) & $\begin{array}{c}\text { Proceso asociado a la } \\
\text { comunicación (PAC) }\end{array}$ & $\begin{array}{c}\text { Se refiere al proceso de interacción en el cual se } \\
\text { expone establecen juicios, por ello está muy vincu- } \\
\text { lada al razonamiento, los procesos de justificación } \\
\text { y la demostración. }\end{array}$ \\
\hline Al (8) & Análisis de información (Al) & $\begin{array}{c}\text { adea cuenta de los procedimientos que se deben } \\
\text { realizar para argumentar, tales como la reflexión, el } \\
\text { estudio de caso, los debates y la socialización. }\end{array}$ \\
\hline
\end{tabular}

A continuación, se presentan algunas definiciones realizadas por los profesores con relación a la argumentación:

Un proceso complejo que permite recopilar información sobre un hecho, un evento, fenómeno o una situación determinada. Es una metodología de enseñanza y aprendizaje que posibilita el conocimiento de la realidad y la búsqueda de soluciones a problemas determinados. (P5).

"Es la justificación convencional ante situaciones específicas (P3)".

"Es un procedimiento discursivo donde se fundamenta una opinión o posición respecto a un tema. (P13)".

"Es una estrategia en la que los estudiantes pueden expresar de forma oral o escrita sus opiniones o ideas sobre un tema en particular, la misma ayuda a determinar el nivel de juicio y razonamientos de los mismos a la hora de opinar (P25)".

A partir de estos resultados, se puede observar que la argumentación como estrategia para el desarrollo de competencias está relacionada con el análisis de información; el trabajo que se realiza en equipo para tomar decisiones; la búsqueda y presentación de datos y pruebas disponibles; es un proceso complejo asociado a la comunicación y que, a su vez se relaciona con la justificación y el razonamiento. Dichos resultados se vinculan con la definición realizada por Osborne (2012), quien la concibe como una actividad verbal, social, racional dirigida a convencer de manera razonable con argumentos. Y, a la vez, con la definición de Aldana (2014) en la cual se destaca el papel de este procedimiento como proceso que genera comunicación entre actores y prácticas de interacción en el proceso educativo y la posibilidad que brinda a los sujetos la capacidad de argumentar, confrontar y justificar según los datos disponibles. De igual manera, se relacionan con los aportes de Toulmin, Rieke, y Janik (1979), quienes expresan la importancia de enseñar actitudes críticas a partir de procesos de razonamiento y argumentación.

En cuanto al segundo objetivo, cuantificar el nivel de importancia que otorgan los docentes a las estrategias de argumentación para el desarrollo de las competencias fundamentales, el $91 \%$ de los docentes admite que las mismas juegan un papel muy importante y solo un 9\% la considera importante, tal como se muestra en la Figura 1.

Figura 1. Valoración sobre la importancia de las estrategias de argumentación

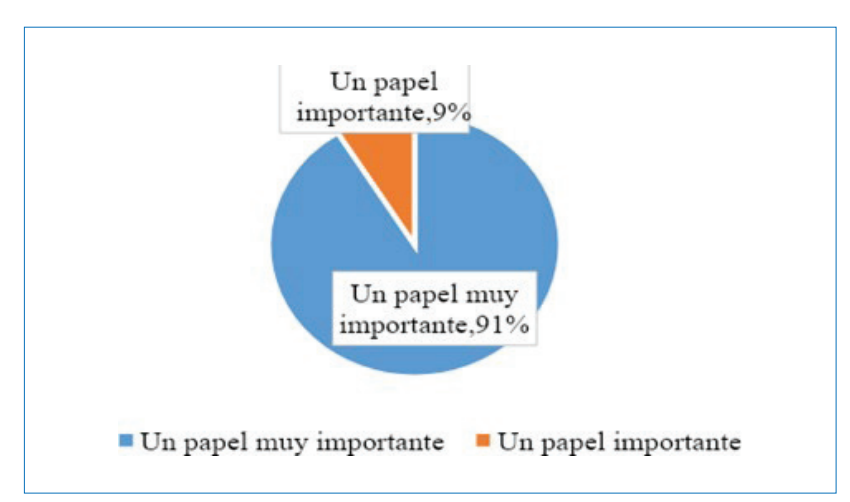


Para el objetivo 3, centrado en las creencias y pensamientos que posee el profesorado sobre las ventajas que aporta esta estrategia en sus prácticas, los resultados destacan que el $85 \%$ de los profesores afirma que la más significativa es promover habilidades, destrezas y capacidades para convencer de manera razonable y lógica con sus argumentos apoyados y sustentados en datos, seguida con un $41 \%$, que ve la ventaja de exponer sus puntos de vista al permitir la participación en debates y foros. Otro 38\% de los docentes consideró ventajoso favorecer el desarrollo de habilidades y destrezas para indagar, hacerse preguntas, realizar hipótesis, buscar información en diversas fuentes, analizar y reflexionar datos, comprobar resultados y dar conclusiones basadas en datos. Cabe destacar que solo un 3\% consideró que una de las ventajas más significativas en la implementación de la estrategia de argumentación es la "construcción de un sujeto racional, lo que fomenta tanto la construcción rigurosa de conocimientos como la formación de ciudadanos democráticos" (ver Figura 2).

Figura 2. Ventajas significativas de la argumentación

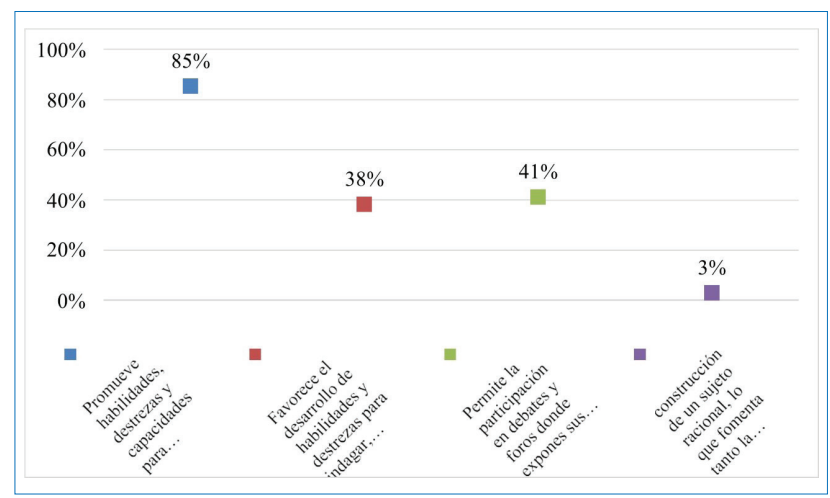

Tales resultados constatan de manera general que el profesorado del ISFODOSU considera que la argumentación como estrategia es muy importante y que la misma se debe a que una de las ventajas más significativa es que promueve habilidades, destrezas y capacidades para convencer de manera razonable y lógica con sus argumentos apoyados y sustentados en datos. Resultados que bien apoyan los diferentes argumentos expuestos por González (2015); González y Pérez (2012) citando al Programa ECBI; Gallego y Márquez (2015) y Osborne (2012).

\section{Conclusiones y recomendaciones}

Con relación a las concepciones de los docentes del ISFODOSU acerca de la estrategia de argumentación para el desarrollo de competencias fundamentales en la formación docente, se pudo observar que los docentes comprenden de manera general en qué consiste la estrategia de argumentación y la vinculan con la búsqueda de datos, razonamientos, justificación y establecimiento de juicios, así como procesos asociados a la comunicación.

En términos generales, los docentes que respondieron el cuestionario consideran que las estrategias de argumentación son procesos complejos y coinciden en afirmar que juegan un papel muy importante para el desarrollo de las competencias fundamentales ya que una de sus ventajas es que favorece el desarrollo de habilidades, destrezas y capacidades para convencer de manera razonable y lógica con sus argumentos apoyados y sustentados en datos.

A partir de los resultados, se recomienda que para el desarrollo e implementación de metodologías que promuevan la argumentación como una estrategia que desarrolle las competencias fundamentales, se debe partir de las características de los estudiantes, el contexto y los resultados de aprendizaje. Además de motivar a los docentes a ampliar sus conocimientos frente a la definición de la argumentación, comprenderla en su totalidad, y posteriormente ser implementada de manera efectiva en los espacios de formación de los futuros docentes del país.

Teniendo en cuenta la importancia de la estrategia de argumentación para el desarrollo de competencias fundamentales, tal como lo plantean los docentes del ISFODOSU que participaron en la investigación, se sugiere fortalecer la implementación de esta estrategia en las diferentes actividades de formación académica en las cuales participan los docentes en formación, así como capacitar a los docentes para definir el rol que deben asumir en el desarrollo de la misma, tal como plantea Conner et al. (2014). Consignas bien definidas, contextos adecuados y las intervenciones de los docentes constituyen los elementos clave para mejorar la capacidad de argumentación de los estudiantes, en este caso, de los futuros docentes. 
Agradecimientos

El equipo de investigación agradece el apoyo financiero recibido por parte de CLACSO e ISFODOSU para la realización de la presente investigación PI-018-2019.

\section{Referencias bibliográficas}

Aldana, E. (2014). La argumentación como estrategia de enseñanza y de aprendizaje de las matemáticas-The argumentation like strategy of education and of learning of the mathematics. Revista científica, 3(20), 37-45. https://revistas.udistrital.edu.co/index.php/ revcie/article/download/7687/9477

Buitrago Martín, Á. R., Mejía Cuenca, N. M. y Hernández Barbosa, R. (2013). La argumentación: De la retórica a la enseñanza de las ciencias. Innovación educativa, 13(63), 17-39. https://n9.cl/c96r

Conner, A. M., Singletary, L., Smith, R. C., Wagner, P. A. y Francisco, R. T. (2014). Teacher support for collective argumentation: A framework for examining how teachers support students' engagement in mathematical activities. Educational Studies in Mathematics, 86, 401-429. https://link.springer.com/article/10.1007/s10649-014-9532-8

Córdoba, A., Ortega, R. y Rodríguez, H. (2005). La formación inicial del profesorado de educación secundaria: un estudio en curso International. Journal of Developmental and Educational Psychology, vol. 2, núm. 1, p. 665. https:// www.redalyc.org/pdf/3498/349832309051. $\underline{\text { pdf }}$

Chehaybar-Kuri, E. y Amador-Bautista, R. (2003). Procesos y prácticas de la formación universitaria. Pensamiento Universitario, 93, 211. http://www.scielo.org.mx/pdf/peredu/ v26n104/v26n104a8.pdf

Cros, A. (2003). Convencer en clase: Argumentación y discurso docente. Ariel. https://ebuah.uah.es/dspace/bitstream/ handle/10017/24720/Convencer Cros LR 2003 01.pdf? sequence $=1$ \&isAllowed $=y$
Crujeiras, B. P. y Jiménez Aleixandre, M. (2012). Competencia como aplicación de conocimientos científicos en el laboratorio: ¿Cómo evitar que se oscurezcan las manzanas? Alambique: Didáctica de las ciencias experimentales, (70), 19-26. http://dx.doi. org/10.5565/rev/ensciencias. 1830

Díaz de Bustamante, J. y Jiménez Aleixandre, M. (1999). Aprender ciencias, hacer ciencias: Resolver problemas en clase. Alambique: Didáctica de las ciencias experimentales, 20, 9-16. https://www.grao.com/es/producto/aprender-ciencias-hacer-ciencias-resolver-problemas-en-clase-al0206011

Gallego, D. y Márquez, F. (2018). La indagación como estrategia para la educación STEAM. Guía Didáctica. https://recursos.educoas.org/ sites/default/files/Final\%200EA\%20Indagacio\%CC\%81n.pdf

González, M. (1995). Formación docente: perspectiva desde el desarrollo del conocimiento y la socialización profesional. Promociones y Publicaciones Universitarias, PPUhttps://jabega.uma.es/discovery/fulldisplay/ alma991003293199704986/34CBUA UMA:VU1

González, C. A. (2015). Concepciones y praxis del profesorado $5^{\circ}$ a $8^{\circ}$ grado de República Dominicana acerca de las prácticas científicas de indagación y argumentación [Trabajo de Fin de Máster no publicado, Instituto Superior de Formación Docente Salomé Ureña].

González, C. A. y Pérez, M. (2012). Manejo de estrategias y recursos para el desarrollo de los procesos pedagógicos en el área de Ciencias de la Naturaleza en el 7mo Grado de la Escuela República de Guatemala [Trabajo de grado, Universidad del país vasco]. https://biblioteca.isfodosu.edu.do/cgi-bin/koha/opac-detail. pl?biblionumber $=17594$ 
Instituto Nacional de Formación y Capacitación del Magisterio (2004). Ordenanza No. 5'2004 que modifica la ordenanza 6'2000 que establece el Reglamento del Instituto Nacional de Formación y Capacitación del Magisterio (INAFOCAM). Santo Domingo, República Dominicana. http:// wbgfiles.worldbank.org/documents/hdn/ed/ saber/supporting doc/LCR/Dominican_Republic/TCH/20130508_075722.pdf

Instituto Superior de Formación Docente Salomé Ureña (2015). Plan de Estudio, Licenciatura en Educación Primaria Segundo Ciclo. Santo Domingo, República Dominicana. http://www. isfodosu.edu.do/images/OfertaAcademica/PerfilEgresados/Perfil\%20Segundo\%20 Ciclo\%20de\%20Primaria.pdf

Jiménez-Aleixandre, M. y Puig, B. (2010). Argumentación y evaluación de explicaciones casuales en ciencias: el caso de la inteligencia. Alambique: Didáctica de las Ciencias Experimentales, 63, 11-18. https:// es.scribd.com/document/468886341/JIMENEZ-ALEIXANDRE-y-PUIG- 2010

Levine, A. (2006). Educating school teachers. Washington:TheEducationSchoolProject. https:// www.researchgate.net/publication/274299906 Stranger Than Fiction Arthur Levine\%27s Educating_School_Teachers--the_Basis_for_a_Proposal

Martínez, M. C. (1999). Hacia un modelo de lectura y escritura: Una perspectiva discursiva e interactiva de la significación. Revista Signos, 32(45-46), 129-147. https:// www.scielo.cl/scielo.php?script=sci arttex$\underline{t \& p i d=S 0718-09341999000100013}$

Ministerio de Educación Superior, Ciencia y Tecnología (2015). Normativa 09-2015, para la Formación Docente de Calidad en la República Dominicana. Santo Domingo, República Dominicana. https://n9.cl/novz4
Ministerio de Educación República Dominicana (2016). Diseño curricular del Nivel Primario del Segundo Ciclo. Santo Domingo, República Dominicana. http://www.ibe.unesco.org/fileadmin/user upload/archive/curricula/dominicanre public/dr upr 2014 spa.pdf

Ministerio de Educación República Dominicana. (2016). Memoria Institucional 2016: Oficina Nacional de Planificación y Desarrollo educativo. Santo Domingo, República Dominicana. http://memorias.minpre.gob.do/api/documents/596/download

Organización para la Cooperación y Desarrollo Económico (2006). Assessing Scientific, Reading and Mathematical Literacy. A Framework for PISA (2006). Programme for International Student Assement. https://n9.cl/5dues

Osborne, J. (2012). The role of argument: Learning how to learn in school science. En B. J. Fraser, K. Tobin, y C. McRobbie (Eds.), Second international handbook of science education. Dordrecht, Hol.: Springer. https://www.researchgate.net/publication/278706715 The Role of Argument Learning How to Learn in School Science

Ruiz, G. (2009). "El enfoque de la formación profesional en torno a la generación de competencia: ¿ejercicio impostergable o "lo que sucedió a un rey con los burladores que hicieron el paño?". Estudios pedagógicos, XXXV (1), 287-299. https://www.scielo.cl/scielo.php?script=sci arttext\&pid=S071807052009000100018

Salazar-Gómez, E. y Tobón, S. (2018). Análisis documental del proceso de formación docente acorde con la sociedad del conocimiento. Revista Espacios, 39(53). http://www.revistaespacios.com/cited2017/ cited2017-17.pdf 
Santana Soriano, E. (2020). Educación por competencias en República Dominicana: perspectiva crítica sobre la práctica. Ciencia y Educación, 4(2), 117-125. https:// revistas.intec.edu.do/index.php/ciened/article/view/1715/2249

Sanz, M. P. G. y Pedreño, L. R. M. (2011). La planificación de evaluación de competencias en Educación Superior. Revista electrónica interuniversitaria de formación del profesorado, 14(1), 113-124. https://dialnet.unirioja.es/ descarga/articulo/3678782.pdf

Serrano de Moreno, S. (2008). Composición de textos argumentativos: Una aproximación didáctica. Revista de Ciencias Sociales, 14(1). http://ve.scielo.org/scielo.php?script=sci arttext\&pid=S1315-95182008000100013\&Ing=es\&nrm=iso\&tlng=es

Tobón, S. (2006). Aspectos básicos de la formación basada en competencias. Talca: Proyecto Mesesup. https://www.uv.mx/rmipe/ files/2019/07/Aspectos-basicos-de-la-formacion-basada-en-competencias.pdf

Toulmin, S.E. (1958). The uses of argument. Cambridge: Cambridge University Press http://johnnywalters. weebly.com/uploads/1/3/3/5/13358288/toulmin-the-uses-of-argument 1.pdf

Toulmin, S., Rieke, T. y Janik, A. (1979). An introduction to reasoning. New York: Macmillan. Versión de Carlos Gutiérrez, C. Virtual Books. http://www.geocities.com/prolenguaje/ elemargumtoul.html

Zabalza, M. Á. y Beraza, M. Á. Z. (2003). Competencias docentes del profesorado universitario: calidad y desarrollo profesional. Narcea ediciones, (4), 7-18. http://www.untumbes. edu.pe/vcs/biblioteca/document/varioslibros/ Competencias\%20docentes\%20del\%20profesorado\%20universitario.\%20Calidad\%20 y\%20desarrollo\%20profesional.pdf

Anexo. Cuestionario:

Concepciones del profesorado del Instituto Superior de Formación Docente Salomé Ureña (ISFODOSU) acerca de las estrategias de indagación y argumentación para el desarrollo de las competencias fundamentales en la formación de maestros.

Enlace para acceder al cuestionario: https:// drive.google.com/file/d/1MvirrWVhTB5Ec4q gMQU5UKH7SDJ9GUQ/view?usp=sharing 